\title{
Análise retrospectiva da prevalência e do perfil epidemiológico dos pacientes vítimas de trauma em um hospital secundário
}

\author{
Retrospective analysis of the prevalence and epidemiological profile of trauma \\ patients in a secondary hospital
}

\author{
Larissa Aparecida Pereira da Silva1, Ariela Carrijo Ferreira', Ruth Elisa Sued Paulino', \\ Gabriela de Oliveira Guedes ${ }^{1}$, Maria Eugênia Benevides da Cunha ${ }^{1}$, \\ Vitória Torrano Carvalho Pereira Peixoto ${ }^{1}$, Talitha Araújo Faria ${ }^{2}$
}

\begin{abstract}
Silva LAP, Ferreira AC, Paulino RES, Guedes GO, Cunha MEB, Peixoto VTCP, Faria TA. Análise retrospectiva da prevalência e do perfil epidemiológico dos pacientes vítimas de trauma em um hospital secundário / Retrospective analysis of the prevalence and epidemiological profile of trauma patients in a secondary hospital. Rev Med (São Paulo). 2017 out.-dez.;96(4):246-54.
\end{abstract}

RESUMO: O trauma consiste em um abalo físico de grande impacto resultante de uma ação abrupta ou violenta que causa danos de extensão variada no organismo, sendo considerado a principal causa de morte nas primeiras quatro décadas de vida. O presente estudo teve como objetivo fazer um levantamento dos dados dos pacientes traumatizados hospitalizados no hospital público de Paracatu-MG, para estabelecer prevalência dos tipos de trauma, caracterizar o perfil epidemiológico das vítimas, indicar as causas mais frequentes, definir a letalidade e informar o tempo de hospitalização. Trata-se de um estudo descritivo transversal retrospectivo baseado na análise de todos os prontuários de pacientes traumatizados do Hospital Municipal de Paracatu-MG, no período de janeiro a dezembro no ano de 2014, constituindo um universo de 544 prontuários. Os resultados apontaram que o mês de dezembro foi o mês com o maior número de traumatizados. Houve maior prevalência no sexo masculino na faixa etária de 20-29 anos. Verificou-se que a principal causa dos traumatismos foi acidente por queda, a segunda causa mais relatada foi acidente de trânsito. A violência foi a terceira maior causa, sendo que as agressões com arma de fogo tiveram o percentual mais significativo. Os traumas ortopédicos constituíram o traumatismo mais prevalente. O maior coeficiente de letalidade foi de $24 \%$, que correspondia ao TCE grave. Conclui-se que há um alto número de casos de trauma em Paracatu-MG, o que evidencia a necessidade de elaboração medidas de prevenção de acidentes domésticos, investimentos em programas de educação no trânsito e melhoria da segurança pública.

Descritores: Ferimentos e lesões/epidemiologia; Hospitalização; Registros médicos; Acidentes por quedas; Acidentes de trânsito; Violência; Brasil/epidemiologia.
ABSTRACT: The trauma consists of a physical shock of great impact resulting from an abrupt or violent action that causes damages of varying extent in the body and is considered the main cause of death in the first four decades of life. The present study aimed to collect data on trauma patients hospitalized at the public hospital of Paracatu-MG, in order to establish the prevalence of type of traumas; to characterize the victim is epidemiological profile and the main causes of the disease, to define the lethality and to inform the duration of hospitalization. This is a cross-sectional retrospective study based on the analysis of 544 medical records of traumatized patients of the Municipal Hospital of Paracatu-MG, from January to December in 2014. The results showed that the month of December was the month with the highest number of traumatized patients. There was a higher prevalence among males aged between $20-29$ years. It was verified that the main cause of the injuries was accident by fall, the second most related cause was traffic accident. Violence was the third largest cause, gunshot assaults having the most significant percentage. Orthopedical traumas were the most prevalent trauma. The highest coefficient of lethality was $24 \%$, which corresponded to severe TBI. It is concluded that there are a high number of trauma cases in Paracatu-MG, which demonstrates the need to measures to prevent domestic accidents, investments in traffic education programs, and improvement of public safety.

Keywords: Wounds and injuries/epidemiology; Hospitalization; Medical records; Accidental falls; Accidentes, traffic; Violence; Brazil/epidemiology.

1. Faculdade de Medicina Atenas, Paracatu, MG, BR. Acadêmicas do $4^{\circ}$ ano do Curso de Medicina. Email: larissaaparecidap@gmail.com, arielacarrijo8@gmail.com, ruthelisasued@hotmail.com, gabi.oliveira.1@hotmail.com, mariaeugeniabene@gmail.com, vitoriatorrano@hotmail.com. 2. Faculdade de Medicina Atenas, Paracatu, MG, BR. Docente do Curso de Medicina. Email: talithabio@yahoo.com.br.

Endereço para correspondência: Larissa Aparecida Pereira da Silva Instituição: Faculdade Atenas. Rua Bernardo Caparucho Melo Franco, 331. Bela Vista, Paracatu, MG. Email: larissaaparecidap@gmail.com 


\section{INTRODUÇÃO}

$\mathrm{O}$ trauma consiste em um abalo físico de grande impacto resultante de uma ação abrupta ou violenta que causa danos de extensão variada no organismo $^{1}$. O grau de complexidade deve ser identificado para determinar a conduta terapêutica do paciente ${ }^{2}$. Na atualidade, o trauma é a principal causa de morte nas primeiras quatro décadas de vida. Afere-se que no ano 2000, o número de mortes tenha sido superior a cinco milhões em todo o mundo ${ }^{3}$.

As consequências econômicas e sociais do trauma são de alta magnitude, devido ao elevado custo da recuperação e gastos da previdência social em situações em que há incapacidade temporária ou permanente da vítima ${ }^{4}$. Além disso, a qualidade de vida do paciente e dos familiares é prejudicada não somente pelos agravos físicos, mas também pelas alterações nas relações sociais, no estilo de vida, na modificação da imagem corporal e distúrbios psicológicos 5 .

Os acidentes automobilísticos e a violência compreendem as principais causas externas e configuram um sério problema de saúde pública ${ }^{6}$. O desenvolvimento industrial, tecnológico e o crescimento urbano possibilitaram o aumento progressivo destas ocorrências, devido a fatores como aumento da frota de veículos, assim como o uso de arma de fogo e arma branca ${ }^{7}$.

No Brasil, o aumento de morte por causas externas ocorreu a partir de 1980, todavia somente em 1997 que os dados provenientes de atendimentos no Sistema Único de Saúde passaram a conter códigos relativos a esses agravos ${ }^{8}$. Estas informações são essenciais para o monitoramento destes problemas no país e, por conseguinte elaboração de projetos e programas de prevenção9. Como exemplos de políticas públicas, ressalta-se a Política Nacional para a Redução da Morbimortalidade por Acidentes e Violência, instituída pela portaria $n^{\circ} 737 / \mathrm{GM}$ em 16 de maio de 2001, que prioriza medidas preventivas contra acidentes e violência, além da garantia de tratamento às vítimas destes eventos ${ }^{10}$.

O cenário mundial estimulou o interesse das organizações internacionais pelo tema ${ }^{9}$. Uma das primeiras ações foi o estabelecimento da conscientização sobre a prevenção de acidentes nos anos de 1961 e 1991. A Organização das Nações Unidas, através da resolução publicada em março de 2010, proclamou o período de 2011 a 2020 como a Década de Ações para a Segurança no Trânsito, objetivando a redução de $50 \%$ dos acidentes de trânsito em todo o mundo ${ }^{11}$.

Ações educativas regionais, principalmente entre crianças e jovens, também são fundamentais para a redução do número de traumatismos. Estas intervenções devem ser conjuntas entre profissionais de saúde, o setor público a sociedade civil, para que sejam eficazes na conscientização sobre os agravos ocasionados pelo infortúnio ${ }^{12}$.
Segundo os autores Rasslan e Birolini ${ }^{13}$, o trauma era considerado uma doença negligenciada, pois os investimentos relacionados à prevenção e tratamento eram inversamente proporcionais ao alto índice de traumatismos no país. Muitos avanços foram realizados, como estratégias de prevenção e atendimento precoce, contudo o trauma ainda é rotulado como um grave problema social e de saúde. Portanto, o conhecimento epidemiológico possibilita uma ampliação dos projetos, além do estabelecimento de prioridades e metas.

Neste contexto, o objetivo do presente trabalho é fazer um levantamento da prevalência dos tipos de trauma no município de Paracatu, caracterizar o perfil epidemiológico dos pacientes hospitalizados por este motivo; indicar os mecanismos de trauma e causas mais frequentes; além de informar o tempo de hospitalização das vítimas.

\section{MÉTODO}

Trata-se de um estudo descritivo transversal retrospectivo através do levantamento da totalidade de prontuários completos indexados em arquivos do Hospital Municipal de Paracatu-MG, referentes aos pacientes hospitalizados no período de janeiro a dezembro no ano de 2014, que deram entrada no serviço de urgência e emergência. O universo compreende 544 prontuários, os quais apresentavam o critério de inclusão de descrição de lesão resultante de causas externas. Foram excluídos os prontuários dos pacientes traumatizados que retornaram ao hospital para a realização de procedimentos agendados ou por complicações do quadro clínico apresentado em internação anterior, a fim de evitar a replicação de dados.

A coleta de dados ocorreu no período de 31 de março a 27 de junho de 2016. Os prontuários foram localizados, examinados e aqueles com critério de inclusão foram separados para que as informações a seguir fossem obtidas: sexo, idade, lesão, mecanismo do trauma, tratamento, evolução, mês de internação e tempo de permanência no hospital.

Os dados foram armazenados em forma de tabelas para relacionar as variáveis de interesse e posteriormente realizou se a elaboração de gráficos e tabelas no Excel (2013). Em seguida, para se obter os resultados necessários foram efetuados cálculos de coeficiente de prevalência dos traumas, sexo e faixa etária; percentual dos traumas e etiologia, além do coeficiente de letalidade.

Este estudo foi enviado e aprovado pelo Comitê de Ética e Pesquisa (CEP/Atenas), seguindo os preceitos éticos, sob Certificado de apresentação para Apreciação Ética número 50962215.6.0000.5100.

\section{RESULTADOS}

A partir da análise de 4881 prontuários referentes 
ao ano de 2014, observou-se que neste ano, 544 pacientes vítimas de trauma deram entrada no hospital, o que representa $11,1 \%$ de todas as internações no local do estudo. O coeficiente de prevalência de traumas foi de $6,4 \%$, portanto, o risco de trauma em Paracatu é 6,4 para cada mil habitantes.

O maior número de internações hospitalares ocorreu em março, mês em que foram registrados 438 prontuários, sendo que $9,4 \%$ dos pacientes eram traumatizados. O Gráfico 1 mostra que dezembro foi o mês com o maior número de traumatizados, os quais representaram $16,7 \%$ dos pacientes hospitalizados no mês e $11,6 \%$ de todos os traumas do ano.

Gráfico 1. Percentual de traumas em relação ao total de internações no mês e percentual de traumas no mês em relação ao total do agravo registrado no ano de 2014, no hospital público de Pracatu-MG

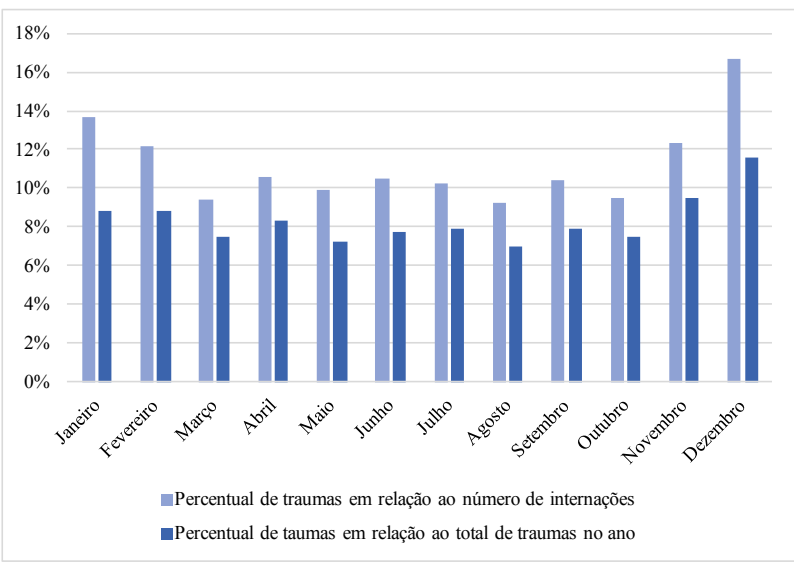

A investigação acerca do perfil epidemiológico dos pacientes traumatizados revelou que em todos os meses do ano de estudo houve maior prevalência no sexo masculino. De acordo com as informações dos prontuários dos pacientes traumatizados, $73,9 \%$ eram do sexo masculino e $26,1 \%$ do sexo feminino. O período com maior número de mulheres vítimas de trauma foi abril, representando $40 \%$ dos hospitalizados no mês (Gráfico 2).

Gráfico 2. Percentual de internações devido trauma por sexo, para o período de 2014, no hospital público de Paracatu-MG

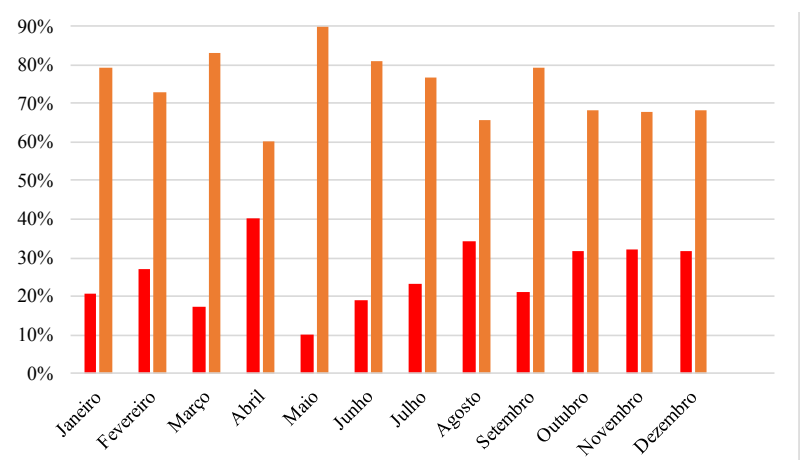

Através da análise da faixa etária dos pacientes hospitalizados pela causa em estudo, notou-se que a maior prevalência de casos ocorreu na faixa etária de 20-29 anos, ou seja, 23,5\%. Tanto o grupo etário que compreende os indivíduos com idade entre 30 e 39 anos quanto aquele com pacientes de 60 anos ou mais, representaram $15,6 \%$ dos casos registrados no ano de 2014. Os pacientes na faixa etária de 13 a 19 anos foram o terceiro grupo mais acometido, com $13 \%$ das internações por trauma. Em seguida, as crianças de 0 a 12 anos representaram 12,8\% dos casos; os adultos de 40 a 49 anos, $11,8 \%$ e a faixa etária de 50 a 59 anos foi a menos acometida com $7,5 \%$ dos casos.

Em relação às etiologias dos traumatismos descritas nos prontuários estudados, verificou-se que $30,5 \%$ destes tinham como causa da injúria uma queda sofrida pelo paciente (Gráfico 3). Segundo os dados analisados, 57,2\% eram do sexo masculino e $42,8 \%$ do sexo feminino e em relação a faixa etária, os idosos foram mais acometidos, pois $27,7 \%$ destes pacientes tinham idade igual ou maior que 60 anos. A segunda faixa etária com maior número de quedas nos registros compreende as crianças de até 12 anos, as quais representam $21,7 \%$ destes pacientes.

A segunda causa mais relatada foi acidente de trânsito, caracterizado pela presença de qualquer tipo de veículo, representando $23,9 \%$ das causas. Os traumas causados por arma de fogo, arma branca e agressão física, representaram respectivamente $11,9 \% ; 2,2 \%$ e $1,6 \%$ dos registros. Os acidentes de trabalho foram a etiologia de $3,5 \%$ dos casos. Entretanto, $21,1 \%$ dos prontuários não possuíam a causa relatada (Gráfico 3).

Gráfico 3. Percentual de traumas por etiologia para o período de 2014, no hospital público de Paracatu-MG

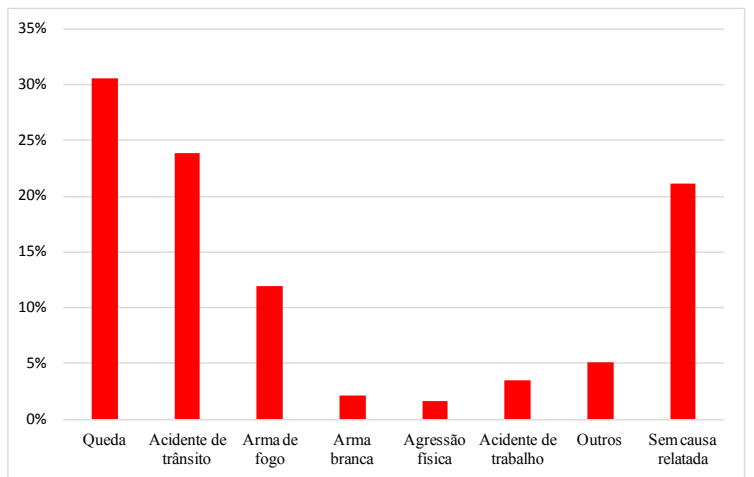

Segundo os dados coletados, $20 \%$ das vítimas de acidentes com veículos que resultaram em lesões traumáticas eram mulheres e $80 \%$ homens, sendo que a faixa etária mais prevalente foi de 20-29 anos representando $34,6 \%$. Os adolescentes de 13 a 19 anos e os adultos de 30 a 39 anos de idade tiveram o mesmo número de casos, representando $15,4 \%$ dos acidentes de trânsito cada. Os adultos de 40 a 49 anos representaram 13\% das vítimas de acidente de transporte terrestre; idosos com 60 anos ou mais representaram 11,5\%; as crianças até 12 anos 5,4\%; 
e o grupo etário de 50 a 59 anos, $4,6 \%$.

Segundo os dados consultados, $95,4 \%$ dos pacientes traumatizados com perfurações por arma de fogo eram do sexo masculino e 4,6\% do sexo feminino. Em relação à faixa etária, $47,7 \%$ destes estavam compreendidos na faixa etária de 20 a 29 anos e $29,2 \%$ entre 13 e 19 anos.

Notou-se que $75 \%$ de todos os pacientes vítimas de perfurações por arma branca eram homens e $25 \%$ mulheres. De acordo com a faixa etária, $41,7 \%$ possuíam idade entre 20 e 29 anos e $16,7 \%$ entre 0 e 12 anos, e a mesma porcentagem na faixa etária de 30 a 39 anos e de 40 a 49 anos. Os pacientes traumatizados que relataram a agressão física, sem uso de armas ou qualquer instrumento, eram todos $(100 \%)$ do sexo masculino, sendo $22,2 \%$ com idade entre 20 e 29 anos e o mesmo percentual para as faixas etárias de 30 a 39 anos, de 40 a 49 anos e de 50 a 59 anos, além de $11,1 \%$ com idade igual ou maior que 60 anos.

Segundo os dados informados nos prontuários, $94,7 \%$ dos pacientes que informaram o acidente de trabalho como causa das lesões eram do sexo masculino e 5,3\% do sexo feminino. Em relação à esta causa, 31,6\% tinham idade entre 30 e 39 anos, 26,3\% entre 40 e 49 anos, $21 \%$ entre 50 e 59 anos, $15,8 \%$ entre 20 e 29 anos e 5,3\% entre 13 e 19 anos.

O Gráfico 4 evidencia o tipo de traumatismo relatado nos prontuários dos pacientes internados. Observa-se que $77 \%$ correspondia a traumas ortopédicos; o trauma torácico representou $7,3 \%$ dos casos; o traumatismo crânio-encefálico (TCE) $6 \%$; o trauma abdominal 3,9\%; o trauma raquimedular, $2,2 \%$ e queimaduras $1,3 \%$. Em $5,5 \%$ dos prontuários o paciente está caracterizado como politraumatizado, ou seja, possui acometimento de dois ou mais sistemas do organismo.

Gráfico 4: Tipos de traumas no ano de 2014, no hospital público de Paracatu-MG

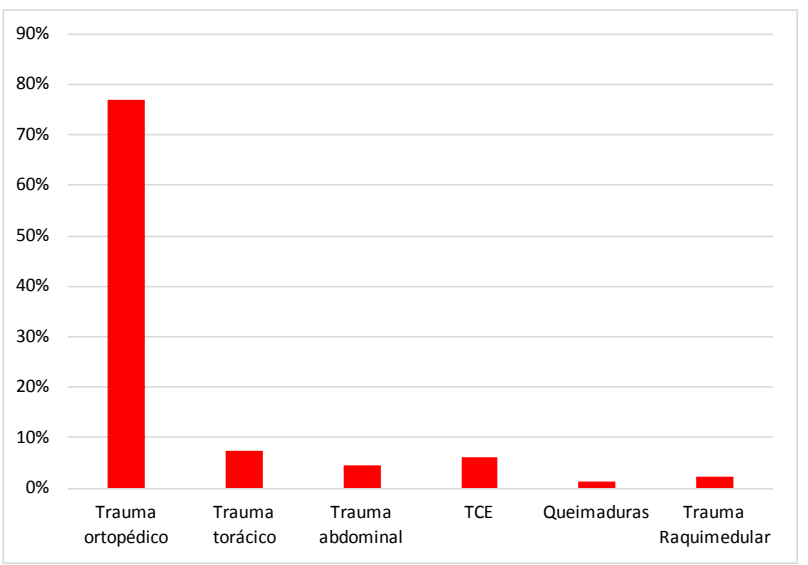

Em relação ao trauma ortopédico, $70 \%$ dos acometidos eram do sexo masculino e $30 \%$ do sexo feminino. A faixa etária na qual há maior número de casos compreende os jovens de 20 a 29 anos, representando
$22,7 \%$ dos casos. Em seguida, os grupos etários, os quais compreendem os pacientes com 60 anos ou mais e os adultos de 30 a 39 anos tiveram o mesmo número de casos, representando 16\% cada. As crianças de 0 a 12 anos representaram $14 \%$ dos casos; os adultos de 40 a 49 anos $11,5 \%$; os adolescentes de 13 a 19 anos, $11,2 \%$ e o grupo de 30 a 39 anos, $8,3 \%$.

Dos traumas ortopédicos, $81 \%$ corresponde a fraturas, 5,2\% correspondem a luxação, 3,3\% lesões tendíneas, 2,9\% amputação traumática, 2,4\% lesão muscular e $5 \%$ não especificado. Em $38 \%$ dos prontuários que relatam fratura, há relatos de que a fratura foi decorrente de uma queda; em $26,4 \%$ o paciente se envolveu em um acidente de trânsito; em $6,9 \%$ a fratura foi uma consequência de um ferimento por arma de fogo; em $2,2 \%$ foi causada por acidente de trabalho e em 1,4\%, foi resultado de uma agressão física. Entretanto, 22,2\% dos prontuários não continham informações sobre a causa do traumatismo. Analisando os casos de fraturas únicas, notouse que $22,3 \%$ consistiam em fratura de rádio, portanto, o osso mais fraturado.

O estudo dos registros evidenciou que 76,8\% dos pacientes com fraturas ósseas sem outros traumas associados foram submetidos à um procedimento cirúrgico, $5,1 \%$ tiveram este tratamento indicado, mas não foi realizado e $2,1 \%$ foram transferidos para outra unidade hospitalar. O coeficiente de letalidade dos casos de fratura é $1,8 \%$, sendo que todos os óbitos foram decorrentes de fratura de fêmur, e destas mortes $83,3 \%$ dos pacientes tinham idade igual ou superior a 60 anos. Conclui-se que o risco de fraturas na população Paracatuense foi de 4,2 em cada mil habitantes no ano de 2014.

Sobre os traumas torácicos registrados nos prontuários, $45 \%$ correspondem a pneumotórax; $35 \%$ a hemopneumotórax; 7,5\% a hemotórax; $5 \%$ a fratura de arcos costais e $2,5 \%$ a trauma cardíaco penetrante e $5 \%$ não especificado. Verificou-se que $90 \%$ dos pacientes eram homens e 10\% mulheres. Em relação a causa, observou-se que $67,5 \%$ dos casos foram consequências de um ferimento por arma de fogo, 22,5\% eram decorrentes de um acidente de trânsito, $5 \%$ originado de um ferimento por arma branca e $5 \%$ de queda. O grupo etário em que este tipo de trauma é mais prevalente é de 20 a 29 anos, o qual corresponde a $42,5 \%$ dos casos. Os adolescentes de 13 a 19 anos representam $20 \%$ dos pacientes hospitalizados por trauma torácico, os adultos de 30 a 39 anos representam 15\%, os idosos com 60 anos ou mais correspondem a $12,5 \%$, o grupo de 40 a 49 anos $5 \%$ e as crianças de até 12 anos e os adultos de 50 a 59 correspondem a $2,5 \%$ cada grupo. O coeficiente de letalidade deste acometimento foi de $12,5 \%$, ou seja, a cada 100 casos, aproximadamente 12 evoluem ao óbito.

Em 50\% dos casos de trauma torácico foi realizado a drenagem pleural, em $42,5 \%$ dos casos foi necessário procedimento cirúrgico e os $5 \%$ restantes correspondem aos casos em que ocorreram os óbitos antes dos procedimentos. 
Segundo os prontuários, $7,5 \%$ dos pacientes foram transferidos para outra unidade hospitalar. O coeficiente de prevalência do trauma torácico no ano de estudo foi $0,47 \%$.

No que se refere ao TCE, $78,8 \%$ dos pacientes eram do sexo masculino e $21,2 \%$ do sexo feminino. Notou-se que a faixa etária mais acometida compreende os idosos com 60 anos ou mais, os quais representaram $30,3 \%$ dos pacientes hospitalizados por essa causa. O segundo grupo etário com mais pacientes com este trauma compreende os indivíduos de 40 a 49 anos, os quais representaram $24,2 \%$. Em seguida, os pacientes adolescentes de 13 a 19 anos representaram $15,1 \%$ dos casos; os jovens adultos de 20 a 29 anos e os adultos de 30 a 39 anos tiveram o mesmo percentual: $12,1 \%$, já as crianças de até 12 anos representaram $6,1 \%$ dos casos.

Em relação as causas do TCE, observou-se que $39,3 \%$ dos pacientes haviam se envolvido em acidente de trânsito; $33,3 \%$ sofreram uma queda; $9,1 \%$ eram vítimas de agressão física; $6,1 \%$ vítimas de ferimentos por arma de fogo; $3 \%$ por arma branca e $6,1 \%$ dos prontuários não apresentavam esta informação. Notou-se que $9 \%$ dos acometidos foram transferidos para outra unidade hospitalar. O coeficiente de letalidade foi de $24,2 \%$, ou seja, a cada 100 pacientes com trauma crânio-encefálico, aproximadamente 24 evoluem a óbito.

No que diz respeito ao trauma abdominal, $88 \%$ dos pacientes eram do sexo masculino e $12 \%$ do sexo feminino. O coeficiente de prevalência deste agravo em 2014 foi de $0,3 \%$. Observou-se que o maior número de casos foi registrado na faixa etária de 13-19 anos, representando $44 \%$ do total de traumas abdominais, em seguida o grupo etário de 20-29 com 40\% dos casos, a faixa de 0-12 anos com 8\% e os indivíduos de 30-39 e maior ou igual a 60 com $4 \%$ cada. Conforme evidenciado nos prontuários, $72 \%$ dos traumas abdominais foram causados por perfurações por arma de fogo, $8 \%$ por arma branca, $12 \%$ por acidentes de trânsito e $8 \%$ por quedas. O coeficiente de letalidade deste trauma foi de $14,3 \%$.

Sobre o trauma raquimedular, $91,7 \%$ dos pacientes eram do sexo masculino e $8,3 \%$ do sexo feminino. Notou-se que $50 \%$ dos pacientes com este tipo de trauma estavam compreendidos na faixa etária de 20 a 29 anos. O segundo grupo mais acometido consiste na faixa etária de 40 a 49 anos, que representou $16,7 \%$ dos casos. A faixa etária de 0 a 12 anos, 13 a 19,30 a 39 e o grupo com idade igual ou superior a 60 anos tiveram o mesmo percentual de casos: $8,3 \%$. Não houve casos entre os pacientes de 50 a 59 anos. Com relação ao mecanismo deste tipo de trauma, constatou-se que $41,7 \%$ se envolveram em acidentes de trânsito, 33\% eram vítimas de perfurações por arma de fogo, $16,7 \%$ sofreram uma queda e $8,3 \%$ dos prontuários não relatavam a causa. Observou-se que $41,7 \%$ das vítimas foram transferidas para outro município e não foram registrados óbitos por esta causa no hospital do estudo.

Em relação aos registros de queimaduras, $71,4 \%$ dos acometidos eram do sexo masculino e $28,5 \%$ do sexo feminino. Em relação a faixa etária, $57 \%$ eram crianças de até 12 anos, $28,5 \%$ adultos de 30 a 39 anos e $14,3 \%$ de 50 a 59 anos. Grande parte dos casos aconteceram na residência do paciente (acidente doméstico), por água quente ou no fogão, sendo estas as causas de $71,4 \%$ das queimaduras. O choque elétrico foi responsável por 14,3\% dos casos. No entanto, esta mesma porcentagem representa os prontuários sem a informação. Não houve óbitos por esta causa no período estudo e $14,3 \%$ foram transferidos para outro município para tratamento em hospital de referência.

Assim como no trauma isolado, a maioria dos politraumatizados são homens, $95,7 \%$, e $4,3 \%$ são mulheres. Em relação a faixa etária, $43,4 \%$ eram jovens de 20 a 29 anos, $21,7 \%$ estavam compreendidos no grupo etário de 13 a 19 anos, $13 \%$ entre $30-39$ anos e em idosos com idade igual ou maior que 60 anos, $4,3 \%$ entre 40 e 49 anos e 50-59 anos.

A maioria dos politraumatizados, $69,5 \%$, foram vítimas de ferimentos por arma de fogo, $26 \%$ sofreram acidente de trânsito e $4,3 \%$ vítimas de agressão física. O coeficiente de letalidade do politraumatismo no ano estudado é de $17,4 \%$. Segundo os prontuários, $13 \%$ foram transferidos para tratamento em outro município.

Através dos dados pesquisados, verificou-se que $28,5 \%$ dos pacientes permaneceram hospitalizados por até 03 dias, $25,7 \%$ de 4 a 07 dias, $28,7 \%$ de 08 a 14 dias, $10,5 \%$ de 15 a 21 dias, $4,2 \%$ de 22 a 28 dias, $0,7 \%$ de 29 a 35 dias, $0,7 \%$ de 35 a 50 dias e $0,5 \%$ permaneceram mais de 50 dias (Gráfico 5).

Gráfico 5. Tempo de internação hospitalar dos traumatizados para o ano de 2014, no hospital público de Paracatu-MG

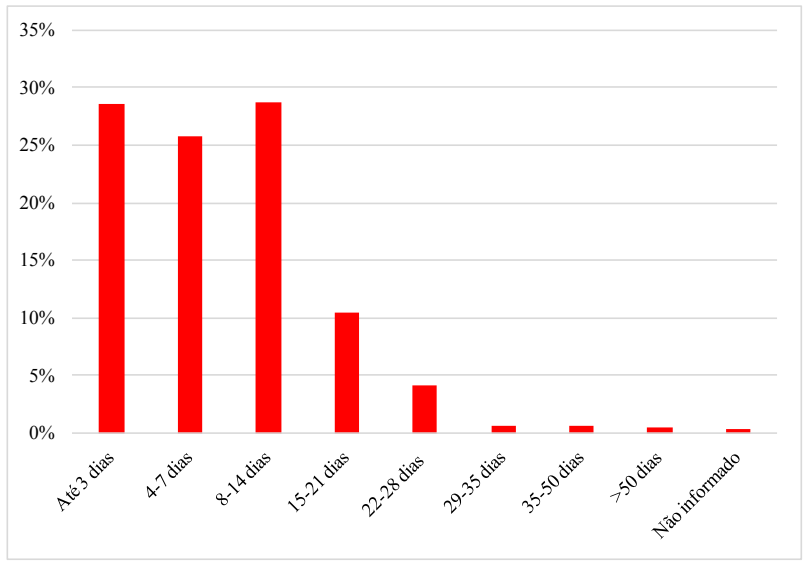

De acordo com os dados obtidos nos prontuários dos pacientes traumatizados ou politraumatizados, $1,8 \%$ evadiram-se do hospital e 4,2\% foram transferidos para tratamento em outra unidade hospitalar. Verificou-se que o coeficiente de letalidade do trauma no período estudado foi de $3,7 \%$, ou seja, a cada cem pacientes aproximadamente 
quatro não sobrevivem. Observou-se que $0,5 \%$ dos prontuários não possuíam informações a respeito da evolução do paciente.

\section{DISCUSSÃO}

O trauma configura um problema de saúde alarmante no país, o que é refletido no município de Paracatu-MG. Nos dados coletados no município, observou-se que no ano de 2014, 544 pacientes vítimas de trauma deram entrada no hospital público, de um total de 4881, o que representa $11,1 \%$ de todas as internações. Este cenário também é encontrado em outros municípios brasileiros, causando graves repercussões na saúde pública, visto que no ano de 2013, um milhão de pessoas foram hospitalizadas por causas externas no país ${ }^{14}$.

Deve-se evidenciar que a metodologia utilizada no estudo pode subestimar os resultados obtidos, visto que as informações foram coletadas em prontuários completos, preenchidos corretamente, com letra legível que apresentavam o critério de inclusão. Sendo assim, o número de pacientes traumatizados atendidos e posteriormente internados no hospital público de Paracatu-MG pode ser maior que o número apresentado pela pesquisa, se os dados dos acometidos não foram corretamente inseridos no registro médico. A análise das causas e mecanismos do trauma também foi prejudicada pela falta de informações, já que aproximadamente $21 \%$ dos prontuários apresentavam relato de lesão por causa externa, mas não mencionavam o fator desencadeante da mesma. Portanto, para termos mais confiabilidade nos dados secundários obtidos nos prontuários do hospital, a melhoria na qualidade de preenchimento dos registros deve ser uma prioridade.

A pesquisa realizada gerou resultados que corroboram com demais estudos da área. Na cidade de Paracatu, durante um ano, a maioria das ocorrências relacionadas ao trauma foram em homens $(73,9 \%)$, confirmando o que demonstram Nogueira e Oliveira ${ }^{15}$, cujo estudo relatou que entre os meses de janeiro a junho no município de Rio Branco-AC, a grande maioria das vítimas é do sexo masculino $(71,2 \%)$. Este fato pode ser relacionado à construção social e à representação histórica da população masculina que corroboram a levá-los a situações de risco ${ }^{16}$.

No presente estudo, constatou-se que a queda foi o mecanismo causador do trauma mais citado nos prontuários $(30,5 \%)$. Frequentemente, as quedas ocorrem devido a soma de vários fatores de risco ou agentes causais, como senilidade ou infância, atividades laborais, uso de medicamentos e morbidades preexistentes. No entanto, este tipo de trauma frequentemente é negligenciado por ser um mecanismo de baixa energia cinética, todavia pode gerar lesões graves e até letais, além de incapacidade posterior.

Os adultos jovens de 20 a 29 anos constituem o grupo etário mais acometido por traumatismos na cidade do estudo. De acordo com a literatura acerca do tema, as causas externas são, em todo o mundo, uma das principais causas de morte entre indivíduos de 15 a 44 anos de idade ${ }^{16}$. Os jovens constituem o grupo etário mais envolvido em acidentes de transporte terrestre, fato associado a condições inseguras, como o excesso de velocidade, embriaguez, comportamento mais agressivo e despreparo ou inexperiência, além do envolvimento em situações de violência e uso de álcool e outras drogas ${ }^{17}$.

A violência em Paracatu-MG é preocupante, uma vez que ocupa a $307^{\mathrm{a}}$ posição no ranking dos municípios mais violentos do país com os maiores índices de homicídios, além de ser o $9^{\circ}$ município de Minas Gerais com a maior taxa de homicídios por arma de fogo, dados do Mapa da Violência, publicado em $2014^{18}$. Esta realidade é evidenciada neste estudo, pois é a terceira causa de traumas atendidos no hospital púbico do município, sendo que o principal mecanismo de trauma envolvido na violência é o ferimento causado por arma de fogo.

Os traumas ortopédicos constituem o trauma mais frequente no hospital público de Paracatu-MG (76,8\%). Assim como os outros tipos de traumas, há predomínio masculino entre os pacientes que sofreram algum tipo de fratura, sendo que $69,7 \%$ eram homens e $30,3 \%$ mulheres. $\mathrm{Na}$ literatura relacionada ao tema também se verifica o maior acometimento de homens, como no estudo feito em um hospital público do Rio de Janeiro-RJ ${ }^{19}$ e em Joaçaba$\mathrm{SC}^{20}$, onde $54,7 \%$ e $72,2 \%$ eram do sexo masculino, respectivamente.

Em relação ao mecanismo do trauma ortopédico caracterizado por fratura, as quedas foram as principais desencadeadoras das lesões, além de constituírem a causa mais frequente na literatura ${ }^{19,20,21}$. Analisando as descrições das fraturas registradas nos prontuários, notou-se que houve uma maior prevalência de acometimento dos membros superiores, principalmente fratura do rádio, o que pode sugerir uma consequência da reação de proteção de apoio da mão ao solo durante a queda ${ }^{21}$.

Alguns estudos discordam quanto à faixa etária mais envolvida em fraturas, que neste estudo, a mais prevalente foi de jovens de 20 a 29 anos, assim como na análise dos traumas em geral, representando $23,9 \%$ dos casos, enquanto que nos escritos de Machado e Duarte ${ }^{19}$ os resultados mostraram que idosos foram maioria nos atendimentos.

No hospital público de Paracatu, os grupos etários referentes aos idosos e às crianças representaram $16 \% \mathrm{e}$ $14 \%$ dos traumas ortopédicos respectivamente, o que está relacionado aos fatores de risco que predispõe às quedas e peculiaridades em relação à terapêutica. A análise das fraturas em idosos, permite concluir que o sexo feminino é mais acometido, o que é confirmado por outros estudos ${ }^{21}$ e pode ser explicado pelo estado funcional mais debilitado e maior morbidade, como a osteoporose, além de maior exposição às atividades domésticas. Quase a totalidade de óbitos decorrentes de fraturas $(83,3 \%)$ ocorreram em idosos 
vítimas de fratura de fêmur devido à queda da própria altura, ademais este tipo de fratura pode gerar a redução da capacidade funcional do idoso mesmo após o tratamento, o que reforça a necessidade de medidas preventivas de queda entre os idosos.

O perfil epidemiológico dos pacientes vítimas de trauma torácico hospitalizados em Paracatu-MG é coerente com o encontrado na literatura ${ }^{22,23,24}$. A faixa etária com o maior número de casos de trauma de tórax compreende os jovens de 20 a 29 anos, assim como encontrado no trauma em geral deste estudo, e em outros estudos publicados como o realizado no Serviço de Emergência do ProntoSocorro Geral do Hospital da Irmandade da Santa Casa de Misericórdia de São Paulo em 2006 ${ }^{22}$, onde houve maior prevalência de homens nesta faixa etária.

Em relação ao tipo de trauma torácico, o penetrante foi mais prevalente, assim como em outros estudos ${ }^{22,23}$. Entretanto há discordância em relação ao mecanismo da lesão, já que os ferimentos por arma de fogo foram mais frequentes (67,5\% dos traumas de tórax) no hospital público de Paracatu, bem como na pesquisa realizada no Hospital Universitário Evangélico de Curitiba ${ }^{24}$, o que difere de outros estudos realizados em São Paulo-SP e São Luís-MA, onde os ferimentos por arma branca foram mais comuns. A causa mais frequente de trauma fechado no hospital do presente estudo foi acidente automobilístico (22,5\% dos traumas torácicos), o que também é coerente com estudos anteriores em outras localidades ${ }^{24}$.

As injúrias mais frequentes no trauma torácico são decorrentes do acúmulo de ar ou sangue no espaço pleural, o que foi confirmado no hospital de estudo: pneumotórax (45\%), hemopneumotórax (35\%) e hemotórax $(7,5 \%)$. Assim como relatado na literatura, o procedimento mais realizado foi a drenagem de tórax. A letalidade deste tipo de trauma em Paracatu foi de 12,5\%, valor intermediário aos coeficientes encontrados nos estudos realizados em Curitiba e São Paulo, 17,3\% e 8,3\%, respectivamente. Segundo o ATLS $^{3, \text { o }}$ diagnóstico e terapêutica imediatas são essenciais para evitar a morte nesse tipo de trauma, no qual a hipóxia, hipercapnia e acidose metabólica são consequências frequentemente presentes nos casos. $\mathrm{O}$ atendimento inicial consiste em avaliação primária, estabilização dos sinais vitais, avaliação secundária e tratamento definitivo.

No município do estudo, o maior número de casos de TCE ocorreu entre os idosos do sexo masculino. Em relação à faixa etária, os pacientes com 60 anos ou mais representaram $30,3 \%$ dos pacientes hospitalizados por TCE, resultado que difere de pesquisas contidas na literatura ${ }^{28,29}$ nas quais há predominância de adultos jovens. Em relação ao sexo, outras publicações confirmam a prevalência em homens, como no estudo de Gaudêncio e Leão ${ }^{28}$, durante os anos de 1992 a 2009. Quanto ao mecanismo do trauma, os resultados encontrados em Paracatu apontam que 39,3\% dos TCEs foram decorrentes de acidente de trânsito e 33,3\% de quedas, assim como ocorreu em outras localidades ${ }^{25,26}$.
O TCE foi o que apresentou a maior letalidade do estudo, 24,2\%, coeficiente próximo ao do estudo realizado em Santa Catarina em um hospital universitário $(26,9 \%)^{26}$. As lesões encefálicas são primárias quando ocorrem no momento do trauma e secundárias, quando é tardia ao acidente, dependente de fatores intra e extracerebrais ${ }^{26}$. Segundo o ATLS ${ }^{3}$, o objetivo do tratamento do TCE é prevenir a lesão secundária, fornecer oxigênio e manter a pressão arterial adequada para perfusão cerebral. Além do elevado índice de óbito decorrente do TCE grave, muitos sobreviventes apresentam danos que resultam em invalidez ou incapacidade para realização de atividades laborais e até mesmo cotidianas.

Em Paracatu, a prevalência do trauma abdominal é maior no sexo masculino ( $88 \%$ ), fato também evidenciados em outros estudos ${ }^{27}$. Quanto ao mecanismo, a principal causa foi a tentativa de homicídio, sendo $72 \%$ por arma de fogo e $8 \%$ por arma branca. Observa-se que o cenário de criminalidade e violência no município envolve indivíduos jovens, principalmente adolescentes, os quais são as maiores vítimas deste tipo de trauma, representando $44 \%$ dos pacientes. Outros estudos sobre esta injúria, relatam o maior acometimento de adultos, acima de $21 \operatorname{anos}^{27}$. A letalidade do trauma abdominal no hospital do estudo foi de $14,3 \%$, valor próximo do resultado do estudo de Leite et al. $^{28}(12,3 \%)$. A avaliação correta do abdome durante o atendimento inicial ao traumatizado é essencial e a demora em reconhecer lesões intra-abdominais pode levar ao óbito precoce por hemorragia ou tardio por lesões de vísceras ${ }^{3}$.

Observando traumas do tipo queimaduras no hospital público de Paracatu, estas representaram 1,3\% dos traumas no ano de 2014, sendo 71,4\% dos pacientes do sexo masculino. Em relação a faixa etária, $57 \%$ eram crianças de até 12 anos, confirmando o que diz Reis et al. ${ }^{29}$, cujo estudo teve maior prevalência de queimaduras foi em crianças, grande maioria do sexo masculino. $\mathrm{O}$ acometimento deste grupo etário pode ser justificado pela maior exposição ao ambiente doméstico, permeado de riscos, principalmente relacionados às queimaduras com líquidos quentes.

\section{CONCLUSÃO}

O estudo realizado no hospital público de Paracatu-MG permite concluir que o trauma é uma importante causa de atendimento e hospitalização. Conclui-se que houve maior prevalência entre os indivíduos do sexo masculino, compreendidos na faixa etária entre 20 e 29 anos, o que provavelmente está associado aos comportamentos de risco no trânsito e no envolvimento com a violência.

Os acidentes de trânsito corresponderam à segunda causa mais relatada dos traumas. A violência em Paracatu-MG demonstrou-se preocupante, visto que constituiu o terceiro motivo mais frequente. Apesar da faixa etária predominante ser de adultos jovens, notou-se um grande número de adolescentes atingidos por arma de fogo e arma branca. 


\section{REFERÊNCIAS}

1. SBAIT. Sociedade Brasileira de Atendimento Integrado ao Traumatizado (SBAIT). O que é trauma? Disponível em: http://www.sbait.org.br/trauma.php.

2. Pereira Júnior GA, Scarpelini S, Basile-Filho A, Andrade JI. Índices de trauma. Rev Med (Ribeirão Preto). 1999;32(3):23750. doi: http://dx.doi.org/10.11606/issn.2176-7262. v32i3p237-250.

3. American College of Surgeons. Advanced Trauma Life Support: student course manual. 9a ed. Chicago: American College of Surgeons; 2012.

4. Whitaker IY, Gutierrez MGR, Koizumi MS. Gravidade do trauma avaliada na fase pré-hospitalar. Rev Assoc Med Bras. 1998;44(2):111-9. doi: http://dx.doi.org/10.1590/S010442301998000200008.

5. Paiva L, Rossi LA, Costa MCS, Dantas RAS. Experiência do paciente politraumatizado e suas consequências. Rev Latino-Am Enfermagem (Ribeirão Preto). 2010;18(6):1-9. doi: http://dx.doi.org/10.1590/S0104-11692010000600024.

6. Oliveira LR, Jorge MHPM. Análise epidemiológica das causas externas em unidades de urgência e emergência em Cuiabá- Mato Grosso. Rev Bras Epidemiol (São Paulo). 2008;11(3):420-30. doi: http://dx.doi.org/10.1590/S1415790X2008000300009.

7. Lyn-Sue J, Siram S, Williams D, Mezghebe H. Epidemiology of trauma deaths in an urban level-1: trauma center predominantly among African Americans implications for prevention. J Natl Med Assoc. 2006;98(12):1940-44. Available from: https://www.ncbi.nlm.nih.gov/pmc/articles/ PMC2569687/pdf/jnma00199-0060.pdf.

8. Gawryszewewski VP, Koizumi MS, Jorge MHPM. As causas externas no Brasil no ano 2000: comparando a mortalidade e a morbidade. Cad Saúde Pública (Rio de Janeiro). 2004;20(4):995-1003. doi: http://dx.doi.org/10.1590/S0102$311 \times 2004000400014$.

9. Jorge MHPM, Gotlieb SLD, Laurenti R. O sistema de informações sobre mortalidade: problemas e propostas para o seu enfrentamento II - Mortes por causas externas. Rev Bras Epidemiol (São Paulo). 2002;5(2):212-23. doi: http://dx.doi. org/10.1590/S1451-790X2002000200008.

10. Ministério da Saúde. Política nacional de redução da morbimortalidade por acidentes e violência. Rev Saúde Pública (São Paulo). 2000;34(4):427-30. doi: http://dx.doi. org/10.1590/S0034-89102000000400020.

11. Organização das Nações Unidas (ONU). Resolução A/64/L.44/Rev.1, de 2 de março de 2010. Proclama a década de ação pela segurança no trânsito - 2011-2020. General Assembly. 2010; Agenda Item 46: 6. Disponível em: http:// www.who.int/roadsafety/news/2014/Final_draft_UN General_Assembly_resolution_improving_global_road safety.pdf

12. Pordeus AMJ, Fraga MNO, Facó TPP. Ações de prevenção dos acidentes e violências em crianças e adolescentes, desenvolvidas pelo setor público de saúde de Fortaleza, Ceará, Brasil. Cad Saúde Pública (Rio de Janeiro). 2003;19(4):1201-03. doi: http://dx.doi.org/10.1590/S0102311 X2003000400041.
13. Rasslan S, Birolino D. O trauma como modelo de doença. Rev Col Bras Cir (Rio de Janeiro). 1998;25(5):3. doi: http:// dx.doi.org/10.1590/S0100-69911998000500001.

14. Brasil. Ministério da Saúde. Secretaria de Vigilância em Saúde. Saúde Brasil 2013: uma análise da situação de saúde e das causas externas. Brasília; 2014.

15. Nogueira JM, Oliveira SMC. Análise epidemiológica das vítimas de trauma. Rev Movimenta (Goiânia). 2014;7(3):73849. Disponível em: www.revista.ueg.br/index.php/ movimenta/article/view/6284.

16. Ferreira MAF, Felizzari CT. Correlação entre a epidemiologia do trauma e $o$ atendimento de enfermagem em uma unidade de pronto-socorro. Rev Eletrônica Fac Evangélica Paraná (Curitiba). 2011;1(1):2-12. Disponível em: http://www. fepar.edu.br/revistaeletronica/index.php/revfepar/article/ view/10/10.

17. Dorigatti AE, Jimenez LS, Redondano BR, Carvalho RB, Calderan TR, Fraga GP. Importância de programa multiprofissional de prevenção de trauma para jovens. Rev Col Bras Cir (Rio de Janeiro). 2014;41(4):245-50. doi: 10.1590/0100-69912014004004.

18. Waiselfisz JJ. Os jovens do Brasil. Mapa da violência. Brasília; 2014. Disponível em: www.mapadaviolencia.org. br/mapa2014_jovens.php.

19. Machado JS, Duarte MS. Incidência de pacientes com fraturas atendidos na emergência de um hospital público na zona oeste da cidade do Rio de Janeiro que realizaram procedimento cirúrgico. Rev FRASCE [online]. 2010. Disponível em: http://www.frasce.edu.br/inativo/frasce/novos_artigos/ incidencia_de_fraturas.pdf

20. Zago APV, Grasel CE, Padilha JA. Incidência de atendimentos fisioterapêuticos em vítimas de fraturas em um hospital universitário. Fisioter Mov (Curitiba). 2009;22(4);56573. Disponível em: www2.pucpr.br/reol/index.php/ RFM?dd1 $=3390 \&$ dd99=pdf.

21. Costa AMR, Xavier EMO, Figueiras MC. Perfil epidemiológico de idosos com fraturas atendidos em hospital de emergência. Rev Bras Ciênc Saúde (São Caetano do Sul). 2012;10(34):41-6. doi: 10.13037/rbcs.vol10n34.1797.

22. Scapolan MB, Vieira NLP, Nitrini SS, Saad Júnior R, Gonçalves R, Perlingeiro JAG, Rivaben JH. Trauma torácico: análise de 100 casos consecutivos. Rev Einsten (São Paulo). 2010;8(3);339-42. doi: http://dx.doi.org/10.1590/s1679$45082010 \mathrm{ao} 1532$.

23. Cuba RMBF, Bezerra JAF. Traumatismo torácico: estudo retrospectivo de 168 casos. Rev Col Bras Cir (Rio de Janeiro). 2005;32(2):57-9. doi: http://dx.doi.org/10.1590/S010069912005000200002 .

24. Naufel Júnior CR, Talini C, Barbier Neto L. Perfil dos pacientes vítimas de trauma torácico atendidos no Hospital Universitário Evangélico de Curitiba (HUEC). Rev Med UFPR (Curitiba). 2014;1(2):42-6. doi: http://dx.doi. org/10.5380/rmu.v1i2.40701.

25. Gaudêncio TG, Leão GM. A epidemiologia do traumatismo crânio-encefálico: um levantamento bibliográfico no Brasil. Rev Neurocienc (São Paulo). 2014;21(3):427-34. doi: 10.4181/RNC.2013.21.814.8p. 
26. Ruy EL, Rosa MI. Perfil epidemiológico de pacientes com traumatismo crânio encefálico. Arq Catarin Med (Florianópolis). 2011;40(3):17-20. Disponível em: http:// www.acm.org.br/revista/pdf/artigos/873.pdf.

27. Lima SO, Cabral FLD, Pinto Neto AF, Mesquita FNB, Feitosa MFG, Santana VR. Avaliação epidemiológica das vítimas de trauma abdominal submetidas ao tratamento cirúrgico. Rev Col Bras Cir (Rio de Janeiro). 2012; 39(4): 302-06. doi: http:// dx.doi.org/10.1590/S0100-69912012000400010.
28. Leite S, Taveira-Gomes A, Sousa H. Lesão visceral em trauma abdominal: um estudo retrospectivo. Acta Med Port. 2013;26(6):725-30. Disponível em: www. actamedicaportuguesa.com/revista/index.php/amp/article/ viewFile/2104/3815.

29. Reis IF, Moreira CA, Costa ACSM. Estudo epidemiológico de pacientes internados na unidade de tratamento de queimados do hospital de urgência de Sergipe. Rev Bras Queimaduras. 2011;10(4):114-8. Disponível em: http://www.rbqueimaduras. com.br/details/80/pt-BR.

Artigo recebido em: 20.02.17

Artigo aceito em: 04.08.17 